\title{
Long-term storage of follicular units in a patient with acute cardiac symptoms during hair transplantation
}

\author{
Karl Stiefsohn, MD, Karl Moser, Claudia Moser, Walter Krugluger, MD Vienna, Austria claudia.moser@moser-medical.com
}

The method of follicular unit storage is critical for the success of the hair transplantation surgical procedure. During the past decade, many attempts have been made to improve the handling of explanted FUs and in formulating new holding solutions that support the metabolic pathways of the isolated FUs. ${ }^{1}$

In our group, attempts have been made to formulate solutions based on tissue culture media. These media clearly demonstrate that FUs stored in improved storage solutions have a higher viability, less apoptotic cells, and an improved growth rate after transplantation. ${ }^{2,3}$ Furthermore, the studies in our lab that evaluated long-term storage of FUs in tissue culture medium-based storage solutions revealed that storage for up to 72 hours is possible, with only slight signs of apoptosis in the stored FUs (Figure 1).

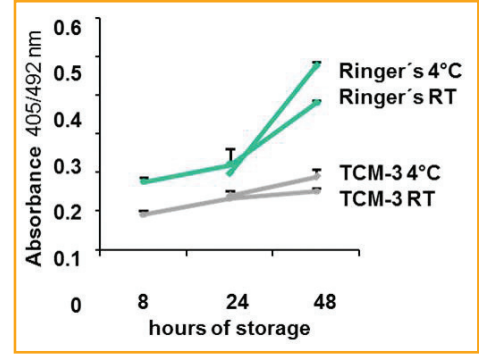

Figure 1. Apoptotic cell death in FUs stored long term in Ringer's solution or in tissue culture medium.

This article reports the routine long-term storage of FUs derived from a patient whose hair transplant session was interrupted due to acute cardiac symptoms.

A hair transplantation session with 1,300 FUs was planned for a 33year-old male patient (Norwood Stage III vertex; Figure 2). In preparation for donor strip removal, the patient was sedated with midazolam $7.5 \mathrm{mg}$

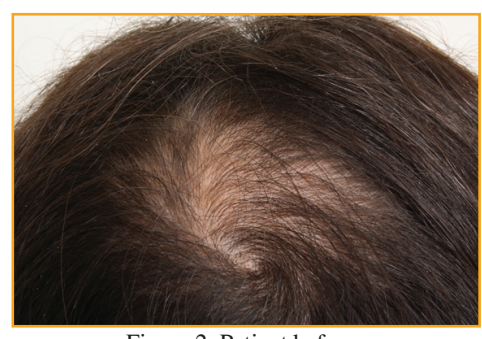

Figure 2. Patient before. and diazepam $5.0 \mathrm{mg}$. Following this an occipital strip $(12.5 \mathrm{~cm} \times 1.5 \mathrm{~cm})$ was removed and the wound was closed using regular methods. After this part of the operation, the patient had a circulatory crisis that resulted in cardiac syncope. In order to stabilize the patient, he was placed in the dorsal position and given 500ml of Ringer's solution intravenously. The patient was then transferred to the hospital's emergency department for further observation and treatment. After hospital admission, he recovered quickly and two days later was able to continue his hair transplantation.

In the intervening time, the donor strip was cut into $0.5 \mathrm{~cm}$-thick slices, which were stored in a modified Williams E medium at $4^{\circ} \mathrm{C}$ under sterile conditions. After 49 hours, the patient returned to continue the hair transplant session (Figure

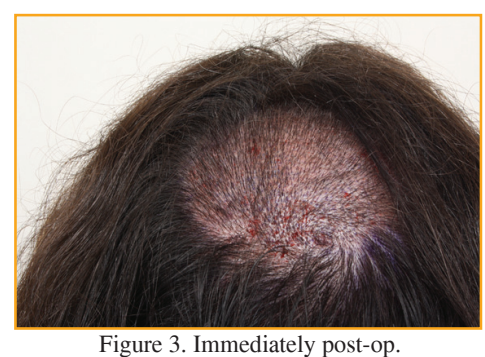

Figure 3. Immediately post-op. 3 ). Even with this delay, no unusual changes in the visible tissue occurred, and the FUs could be prepared using the Mantis Vision engineering microscope. During surgery, the isolated FUs were stored in saline solution and 1,230 FUs were transplanted to the recipient area.

Evaluation of the patient after 3 and 8 days showed a regular course of wound healing and graft integration in the transplanted area. The hair count in the transplanted area 6 months after the procedure revealed regular post-transplantation hair growth (Figure 4).

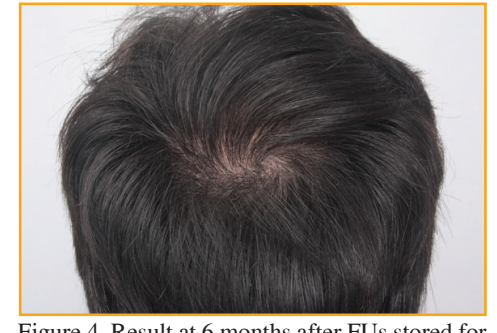

Figure 4. Result at 6 months after FUs stored for 48 hours in Williams E medium at $4^{\circ} \mathrm{C}$.
In the course of transplantation with FUs stored in situ in the donor strip for 48 hours, the usefulness of improved storage conditions for long-term storage of FUs could be demonstrated. No significant differences as compared to the standard storage of FUs were found in this patient; therefore, for unpredictable conditions that could possibly occur during hair transplantation, long-term storage of FUs in appropriate storage solutions is a viable option that results in a very satisfactory outcome for the patient.

\section{References}

1. Mathew, A.J. A review of cellular biopreservation considerations during hair transplantation. Hair Transplant Forum Int'l. 2013; 23(1):1-11.

2. Krugluger, W., et al. Enhancement of in vitro hair shaft elongation in follicles stored in buffers that prevent follicle cell apoptosis. Dermatol Surg. 2004; 30:1-5.

3. Krugluger, W., et al. New storage buffers for micrografts enhance graft survival and clinical outcome in hair restoration surgery. Hair Transplant Forum Int'l. 2003; 13(3):333-334. 